\title{
On Residual Dose Rate Within Particle Accelerator Enclosures
}

\author{
J.D. Cossairt \\ Fermi National Accelerator Laboratory \\ P.O. Box 500, Batavia, Illinois 60510
}

January 1996

Submitted to Health Physics

Formerly published under the title, "Theorem on the Photon Fluence Interior to a Closed Surface of Uniform Specific Emission" 


\section{Disclaimer}

This report was prepared as an account of work sponsored by an agency of the United States Government. Neither the United States Government nor any agency thereof, nor any of their employees, makes any warranty, express or implied, or assumes any legal liability or responsibility for the accuracy, completeness, or usefulness of any information, apparatus, product, or process disclosed, or represents that its use would not infringe privately owned rights. Reference herein to any specific commercial product, process, or service by trade name, trademark, manufacturer, or otherwise, does not necessarily constitute or imply its endorsement, recommendation, or favoring by the United States Government or any agency thereof. The views and opinions of authors expressed herein do not necessarily state or reflect those of the United States Government or any agency thereof. 


\title{
ON RESIDUAL DOSE RATE WITHIN PARTICLE ACCELERATOR ENCLOSURES
}

\author{
J. Donald Cossairt \\ Fermi National Accelerator Laboratory \\ P.O. Box 500 \\ Batavia, IL 60510
}

\begin{abstract}
A mathematical proof of a theorem concerning the flux density of particles at any point interior to a simple closed convex surface in which the surface emits such particles uniformly and isotropically is presented. The relationship of this theorem, which is of rather general validity, to related observations of other workers is discussed. The ramifications of the theorem are considered with particular attention devoted to conditions commonly found at particle accelerators. It is concluded that results of this theorem are directly applicable to efforts to apply the principle of maintaining dose equivalents received by personnel as low as reasonably achievable (ALARA).*
\end{abstract}




\section{INTRODUCTION}

The calculation of radiation fields in the vicinity of a number of solids or surfaces containing radioactive materials has been a subject of many treatises. Such efforts generally have emphasized the calculation of radiation fields due to photon-emitting radionuclides that have resulted from contamination or activation processes. Photon-emitters are popular subjects of such calculations due to the fact that photons, compared with other radiations (i.e., $\beta$ - and $\alpha-$ particles), are relatively more penetrating and are also not subject to Coulomb scattering processes. Neutrons are seldom considered in such calculations since they are not usually emitted in radioactive decay and their interactions with matter are much more complex. The concentrations of the radionuclides in the solids are taken to be either uniform or welldescribed by simple analytical formulae. Examples of such calculations have been presented by Jaeger et al. (1968) and Chilton et al. (1984).

It is clear that such calculations, when performed for situations involving source geometries other than "points" or "infinite lines", can become very difficult due to integrations performed over complicated geometries. Also, most of the calculations reported in the literature are done for points external to the solid containing the radioactivity. This is a natural result of the concern for understanding the spatial dependence of the radiation field in the vicinity of such objects in order to better plan activities such as maintenance operations. In contrast, very few calculations exist which address the flux density of particles or the radiation fields inside cavities within such objects.

At particle accelerators, components of the machines and experimental equipment are typically located within cnclosures. When accelerated particles that have kinetic energies in excess of a few $\mathrm{MeV}$ are present, radioactivity is induced in these components primarily duc to the interaction of neutrons, produced by beam interaction with material, of a variety of energies ranging from the beam energy down to thermal energies. Moreover, the activation due to these neutrons is not confined to the components but includes the enclosure walls as well. One 
needs to understand the activation of the components relative to that of the walls in order to understand the total dose equivalent rate within the room.

Armstrong and Barish (1969) studied this topic for the specific case of the interior of a cylinder concentric with a smaller cylinder of iron. The outer cylinder is representative of the walls of an accelerator enclosure while the inner one is representative of accelerator components such as bending magnets. They demonstrated that if the enclosure walls comprising the outer cylinder is uniformly activated, the photon dose equivalent rate due to the activated walls is a constant independent of position within the cylinder and identically equal to the dose equivalent rate at any point on the wall surface. Their derivation was specific to the interior of a cylindrical enclosure of finite radius but of infinite length. In this paper a proof of a general theorem which confirms this specific result is offered and its ramifications are discussed.

\section{THE THEOREM}

Consider a simple closed convex surface which emits an isotropic flux density of particles, $\phi_{0}\left(\right.$ e.g., $\left.\mathrm{m}^{-2} \mathrm{~s}^{-1}\right)$ that is constant over the inner face of the surface so that the emission from a unit of surface area into any given direction is $\phi_{0} / 4 \pi \mathrm{sr}^{-1}$. Such surfaces are defined precisely in mathematics (e.g., Buck, 1965 or James and James, 1969). Here "simple" means that any closed curve within the volume enclosed by the surface can be shrunk to a point while remaining within the volume. Thus, for example, the surface of a donut is not such a surface. "Closed" means that it has no "holes" while "convex" implies that all pairs of points within the space bounded by the surface can be connected by line segments that lie completely within that space. Fig. 1 illustrates an example of such a surface that includes the origin of coordinates and thus defines a cavity. The surface could be the inner boundary of an activated or contaminated solid or a thin surface that is, itself, activated or contaminated. It is desired to calculate the flux density, $\boldsymbol{\phi}$, at some arbitrary point in space, $\mathbf{P}$, located within the 
cavity bounded by the surface. $\mathbf{P}$ is located at radius vector $\overrightarrow{\mathbf{r}}$. Consider the contributions from the particle flux density emitted by some elemental area $\mathbf{d} \overrightarrow{\mathbf{A}}$ of the surface to the quantity $\boldsymbol{\phi}$ at $\mathbf{P}$. The vector $\mathbf{d} \overrightarrow{\mathbf{A}}$ is defined, as is customary, normal (i.e., perpendicular) and outward to the surface at an arbitrary location on the surface defined by radius vector $\overrightarrow{\mathbf{r}^{\prime}}$. The increment of flux density at point $\mathbf{P}$ due to elemental area $\mathbf{d} \overrightarrow{\mathbf{A}}$ is given by:

$$
d \phi=\frac{\phi_{0}}{4 \pi} \frac{d \vec{A} \cdot \hat{n}}{\left|\overrightarrow{\mathbf{r}^{\prime}}-\vec{r}\right|^{2}},
$$

where the unit vector $\hat{\mathbf{n}}$ is along the vector $\overrightarrow{\mathbf{r}^{\prime}}-\overrightarrow{\mathbf{r}}$ and is given by

$$
\hat{\mathbf{n}}=\frac{\overrightarrow{\mathbf{r}^{\prime}}-\overrightarrow{\mathbf{r}}}{\left|\overrightarrow{\mathbf{r}^{\prime}}-\overrightarrow{\mathbf{r}}\right|}
$$

The scalar product in the numerator of eqn (1) is positive-definite due to the fact that the surface is convex. The solid angle $\mathbf{d} \boldsymbol{\Omega}$ subtended at $P$ by element of surface area $\mathbf{d} \overrightarrow{\mathbf{A}}$ is

$$
\mathbf{d} \Omega=\frac{\mathbf{d} \overrightarrow{\mathbf{A}} \cdot \hat{\mathbf{n}}}{\left|\overrightarrow{\mathbf{r}^{\prime}}-\overrightarrow{\mathbf{r}}\right|^{2}} .
$$

Thus, by inspection,

$$
d \phi=\frac{\phi_{0}}{4 \pi} d \Omega
$$


Performing the integration over the totality of solid angle surrounding $\mathbf{P}$,

$$
\phi=\oint \frac{\phi_{0}}{4 \pi} d \Omega=\frac{\phi_{0}}{4 \pi} \oint d \Omega=\phi_{0} .
$$

This proof is physically valid if particles originating from an elemental area of the surface are not scattered back toward point $\mathbf{P}$ from some other point on the surface, since the latter effect was not considered in the above derivation. Thus, one can state the following theorem:

The flux density of particles at any point interior to a simple closed convex surface in which the surface emits such particles uniformly and isotropically is equal to the flux density of such particles at the surface, provided the scattering of the particles by the surface can be neglected. $\S$

The present theorem is similar to, but not identical with the following theorem proven by Barbier (1969) as a part of his discussion of photon dose equivalent rates in the vicinity of objects of various shapes:

"The radiation field inside a cavity imbedded in an infinite volume of radioactive material of uniform activity is independent of the form of the cavity and constant from point to point therein".

The method of derivation of the present theorem differs from that of Barbier but leads to equivalent results. It essentially starts with a uniformly radioactive surface rather than a uniformly radioactive volume of material surrounding a cavity. The present theorem also clearly covers the case where the induced activity is "buried" in the wall. It merely requires that the flux density at the surface be uniform. 
There is a subtlety associated with the integration of flux density, an inherently scalar quantity. Fig. 2 shows an example of cavity bounded by a more convoluted simple closed surface of uniform and isotropic activation or contamination that is demonstrably not everywhere convex. Consider an element of solid angle $\mathbf{d} \boldsymbol{\Omega}$ shown in Fig. 2. It appears that the contribution of the flux density from all three elements of area $\left(\mathbf{d} \overrightarrow{\mathbf{A}}_{1}, \mathbf{d} \overrightarrow{\mathbf{A}}_{2}\right.$, and $\mathbf{d} \overrightarrow{\mathbf{A}}_{\mathbf{3}}$ ) included in $\mathbf{d} \boldsymbol{\Omega}$ contribute to the flux density at $\mathbf{P}$. Thus this particular element of solid angle would receive three times the flux density as those which only viewed one such surface element $\mathbf{d} \overrightarrow{\mathbf{A}}$ as in Fig. 1. Since flux density is a physical quantity that can only be positive, the contribution $\mathbf{d} \phi$ at $\mathbf{P}$ due to $\mathbf{d} \overrightarrow{\mathbf{A}}_{2}$ involves taking the absolute value of the scalar product in eqn (1) since otherwise a nonphysical value of $\mathbf{d} \phi<0$ results. Such a condition would invalidate the theorem. This problem arises if the surface is a thin sheet that does not attenuate the radiation field. However, if the intervening material outside the surface illustrated in Fig. 2 by the crosshatched material in the element of solid angle $\mathbf{d} \boldsymbol{\Omega}$ between the surfaces $\mathbf{d} \overrightarrow{\mathbf{A}}_{1}$ and $\mathbf{d} \overrightarrow{\mathbf{A}}_{2}$ provides significant absorption of the radiation, then only the inner surface area element, $\mathbf{d} \overrightarrow{\mathbf{A}}_{\mathbf{1}}$, will contribute appreciable flux density to the integral and the theorem represents a valid approximation.II

\section{DISCUSSION}

At accelerators, the principal radiological hazard due to work on activated equipment is from photons of energies up to a few $\mathrm{MeV}$ emitted by radioactivated components and enclosure walls. This residual radioactivity may be produced through the interactions of the direct charged-particle beam. However, a large portion of the activation is due to the copious production of "fast" neutrons (i.e., neutrons with kinetic energies exceeding one $\mathrm{MeV}$ ) by the direct beam. Such neutrons then interact in the components and enclosure walls to also produce residual radioactivity. For photons of energies usually emitted by such activated materials, the albedo is generally small, typically less than five per cent and often less than one 
per cent (Chilton et. al. 1984, NCRP, 1977). Thus the lack of significant scattering is evident and this condition of the theorem is approximately valid.

Furthermore, the accelerator components are nearly always housed in an enclosure comprised of concrete or earth. Most of these enclosures are well approximated by simple closed convex surfaces. This can include long tunnels if they can be considered to be "infinite". Thus another condition of the theorem is commonly approximately met. Likewise, the "convoluted" condition illustrated in Fig. 2 is generally not a problem for accelerator enclosures since "turns" or "bends" in the walls generally involves the presence of intervening material that provides significant attenuation of photons.

Earth or concrete are hydrogenous because of their water content and are thus clearly capable of "thermalizing" the fast neutrons. Such thermal neutrons essentially fill a localized portion of the interior volume of enclosure near a point of beam interaction as a "gas" with an approximately uniform flux density. They thus irradiate the enclosure walls with approximate uniformity over a localized region. The phenomena has been described by Patterson and Thomas (1973). More recently, Ishikawa, et al. (1991) have conducted measurements of the dependence of thermal neutron flux density upon the energy of the neutrons present and upon the location within a concrete room at an accelerator. Their conclusion was that at distances from targets exceeding approximately four meters, the normalized flux density of thermal neutrons is approximately independent of location. A similar result was verified experimentally in a different measurement by Kimura, et al. (1994). Thus, thermal neutrons in an accelerator enclosure provide a possible mechanism for creating conditions of approximate uniform activation of the walls through the thermal neutron capture process.

It has long been known that natural sodium, which is monoisotopic $\left({ }^{23} \mathrm{Na}\right)$, present as a "trace element" in concrete, can be the origin of an important source of occupational dose equivalent because of the large $\left(\sigma_{\text {th }}=0.54 \times 10^{-24} \mathrm{~cm}^{2}\right)$ cross section for the ${ }^{23} \mathrm{Na}(\mathrm{n}, \gamma)^{24} \mathrm{Na}$ thermal neutron capture reaction (Seelmann-Eggebert et al. 1981). This reaction is particularly important to residual dose equivalent rates because of the rather high energies of the two 
$\gamma$-rays, 2.75 and $1.37 \mathrm{MeV}$, that are emitted in 100 and 99.9 per cent, respectively, of the subsequent decays (Browne et al. 1986). Furthermore, the 14.96 hour half-life of ${ }^{24} \mathrm{Na}$ (Seelmann-Eggebert et al. 1981) renders this radionuclide important at accelerators during maintenance operations which must be done promptly (i.e., within a day or so) following cessation of beam operations in order to minimize the length of accelerator shutdowns. This reaction provides a process in which the thermal neutrons can readily produce significant levels of residual radioactivity in a typical accelerator enclosure.

At relatively low kinetic energies, say less $E<50 \mathrm{MeV}$, many of the nuclear reactions that are energetically possible have angular distributions that are relatively isotropic. These can result in the production of radionuclides having spatial distributions that may approximate the uniformity condition of the theorem. At higher energies other processes such as spallation reactions which are decidely forward-peaked and hence anisotropic (see, for example, Barbier, 1969) become important in producing the induced radioactivity and will thus produce distributions of radionuclides that may not meet this uniformity condition. Likewise, walls containing extremely low concentrations of sodium may lead to a situation where the conditions of the theorem are not well-matched due to the minimization of the production of ${ }^{24} \mathrm{Na}$ and consequent reduction in sensitivity to the thermal neutrons. However, at the higher energies, the production of secondary particles in a hadronic cascade, can, in some situations, "smear out" the distribution of radionuclide production in ways that for a localized region may approximately meet the uniform activation condition. Thus the theorem can still be of interest qualitatively at the higher energies.

Armstrong and Barish (1969), in an effort to study the particular problem of ${ }^{24} \mathrm{Na}$ production, have calculated residual dose rates inside a cylindrical accelerator tunnel of infinite length due to both the magnets and the concrete walls for $3 \mathrm{GeV}$ protons incident on an iron target which simulates beam components. ${ }^{\dagger}$ It was concluded by Armstrong and Barish that cven for concrete containing only one per cent sodium by weight, the dose equivalent rate at the surface of the tunnel wall, for an infinitely long irradiation, is dominated by the dose 
equivalent due to the radioactivity in the wall rather than from that due to the radioactivity in the iron target for a period of approximately 30 hours after cessation of beam.

This theorem, even if its conditions are met only in an approximate way, has important consequences with respect to two of the three time-honored principles of keeping radiation dose equivalents as low as reasonably achievable (ALARA). These principles are: the minimization of the time of exposure, the maximization of the distance of the exposed individual from the source, and the employment of appropriate shielding. Under conditions where the theorem is valid, maximizing the distance from the accelerator components to the work location does not reduce the dose equivalent because the latter is not a function of position within the enclosure. Likewise, removal of the accelerator components or shielding them does not lead to the anticipated reduction in dose equivalent rates within the enclosure because of the dominance of the dose equivalent due to the photons emitted by the wall. Knowledge of the phenomenon expressed by the theorem may well lead one to delay work (e.g., maintenance or repair activities) in locations where the conditions of the theorem are likely to approximately met in order to provide for sufficient time for the ${ }^{24} \mathrm{Na}$ to decay. After most of the ${ }^{24} \mathrm{Na}$ has decayed, shielding the accelerator components and/or maximizing the distance between them and the work location will, in conjunction with the minimization of the time of exposure, then be effective in controlling exposures.

\section{CONCLUSION}

A theorem has been proven in a general manner which states that: "The flux density of particles at any point interior to a simple closed convex surface in which the surface emits such particles uniformly and isotropically is equal to the flux density of such particles at the surface, provided the scattering of the particles by the surface can be neglected". Under conditions often found in practical circumstances, the theorem is approximately valid. The theorem has significant ramifications for conditions commonly found at particle accelerators that are directly 
applicable to efforts to apply the principle of maintaining dose equivalents received by personnel as low as reasonably achievable (ALARA). These are:

1) that the shielding of accelerators components may not under some circumstances effectively limit dose equivalents to maintenance personnel, and,

2) that increasing the distance of such workers from accelerator components may not significantly limit the dose equivalent received.

In fact, the radioactivity of the walls of the enclosure itself may, under some conditions, dominate the planning of maintenance work in accelerator enclosures. Even in circumstances where the theorem is not strictly valid, the concept is important in that it leads one to not neglect the importance of the activation of enclosure walls at accelerators relative to the activation of the beam components.

Acknowledgments - The author wishes to thank David Boehnlein, Vernon Cupps, Alexander Elwyn, and Elaine Marshall of the Fermilab staff for their provocative comments on this paper. The author also acknowledges the receipt, some years ago, of insight into the mathematical basis of this theorem from the late Professor Emil J. Konopinski of Indiana University. 


\section{REFERENCES}

Armstrong, T. W; and Barish, J. Calculation of the residual photon dose rate due to the activation of concrete by neutrons from a $3-\mathrm{GeV}$ proton beam in iron. In: Proceedings of the Second International Conference on Accelerator Dosimetry and Experience, Stanford Linear Accelerator Center, November 5-7, 1969. United States Atomic Energy Commission, CONF691101 (TID-4500); 1969: 70-91.

Barbier, Marcel. Induced radioactivity. Amsterdam, Netherlands: North-Holland Publishing Company; 1969: 62-81.

Browne, Edgardo; Firestone, Richard B; Shirley, Virginia S. Table of radioactive isotopes. New York, New York: John Wiley and Sons; 1986: p. 24-2.

Buck, R. Creighton. Advanced Calculus. New York, New York: McGraw-Hill Book Company; 1965.

Chilton, Arthur B.; Shultis, J. Kenneth; Faw, Richard E. Principles of radiation shielding. Englewood Cliffs, New Jersey 07632: Prentice-Hall, Inc.; 1984: 145-186, 274-278.

Ishikawa, Toshio; Sugita, Hiroshi; and Nakamura, Takashi. Thermalization of acceleratorproduced neutrons in a concrete room. Health Phys. 60: 209-221; 1991.

Jaeger, R. G.; Blizard, E. P., Chilton, A. B.; Grotenhuis, M.; Hönig, A.; Jaeger, Th. A.; Eisenlohr, H. H. Engineering compendium on radiation shielding, Volume 1 shielding fundamentals and methods. New York, New York: Springer-Verlag, Inc.; 1968: 363-416. 
James, Glenn; James, Robert C. Mathematics dictionary. Princeton, New Jersey: D. Van Nostrand Company, Inc.; 1959: 380.

Kimura, Ken-ichi; Ishikawa, Toshio; Kinno, Masaharu; Yamadera, Akira; and Nakamura, Takashi. Residual long-lived radioactivity distribution in the inner concrete wall of a cyclotron vault. Health Phys. 67: 621-631; 1994.

Konopinski, Emil J. Electromagnetic fields and relativistic particles. New York, New York: McGraw-Hill Book Company; 1981: 9-12.

National Council on Radiation Protection and Mcasurements. Radiation protection design guidelines for 0.1-100 MeV particle accelerator facilities. Bethesda, Maryland: NCRP Report No. $51 ; 1977: 110$.

Patterson, H. Wade, Thomas, Ralph H. Accelerator health physics. New York, New York: Academic Press; 1973: 513-518.

Seelmann-Eggebert, W; Pfenning, G.; Münzel, H; Klewe-Nebenious, H. Chart of the nuclides. Karlsruhe, Germany: Kernforschungszentrum; 1981. 


\section{List of Figure Captions}

Fig. 1. Coordinate geometry for deriving the relationship between a simple closed convex surface of uniform, isotropic emission and the flux density at any point within it.

Fig. 2. Effect of a convoluted, non-convex surface of uniform, isotropic emission upon the results of this paper. The cross-hatched region could represent a material capable of significant attenuation of the radiation emitted by the surface. 


\section{Footnotes}

* This work was performed at the Fermi National Accelerator Laboratory, which is operated by Universities Research Association, Inc., under contract DE-AC02-76CH03000 with the U. S. Department of Energy.

$\S$ The argument can be intuitively understood as follows. When one looks at an infinite surface within a cone of fixed angular aperture, the apparent brightness of such a surface emitting a uniform flux density is independent of the distance from the observer to the surface because the decrease in intensity due to the inverse square geometry as one moves away from the surface is exactly balanced by the increase in area viewed. This is a fact well know to astronomers, photographers, and artists.

II As readers will recognize, the mathematics involved in this derivation is similar to that found in connection with the Gauss Law of electrostatics. The principal difference is that the Gauss Law is concerned with the integral over the surface is of a vector quantity, the electric field. Negative values of the scalar product in the equivalent to eqn (1) are thus allowed. Because of this fact, the Gauss Law is valid for all simple closed volumes, even ones that are not convex as is shown, for example, by Konopinski (1981).

$\dagger$ These authors have included in their analysis some other reactions such as spallation along with thermal neutron capture which are capable of producing ${ }^{24} \mathrm{Na}$. 


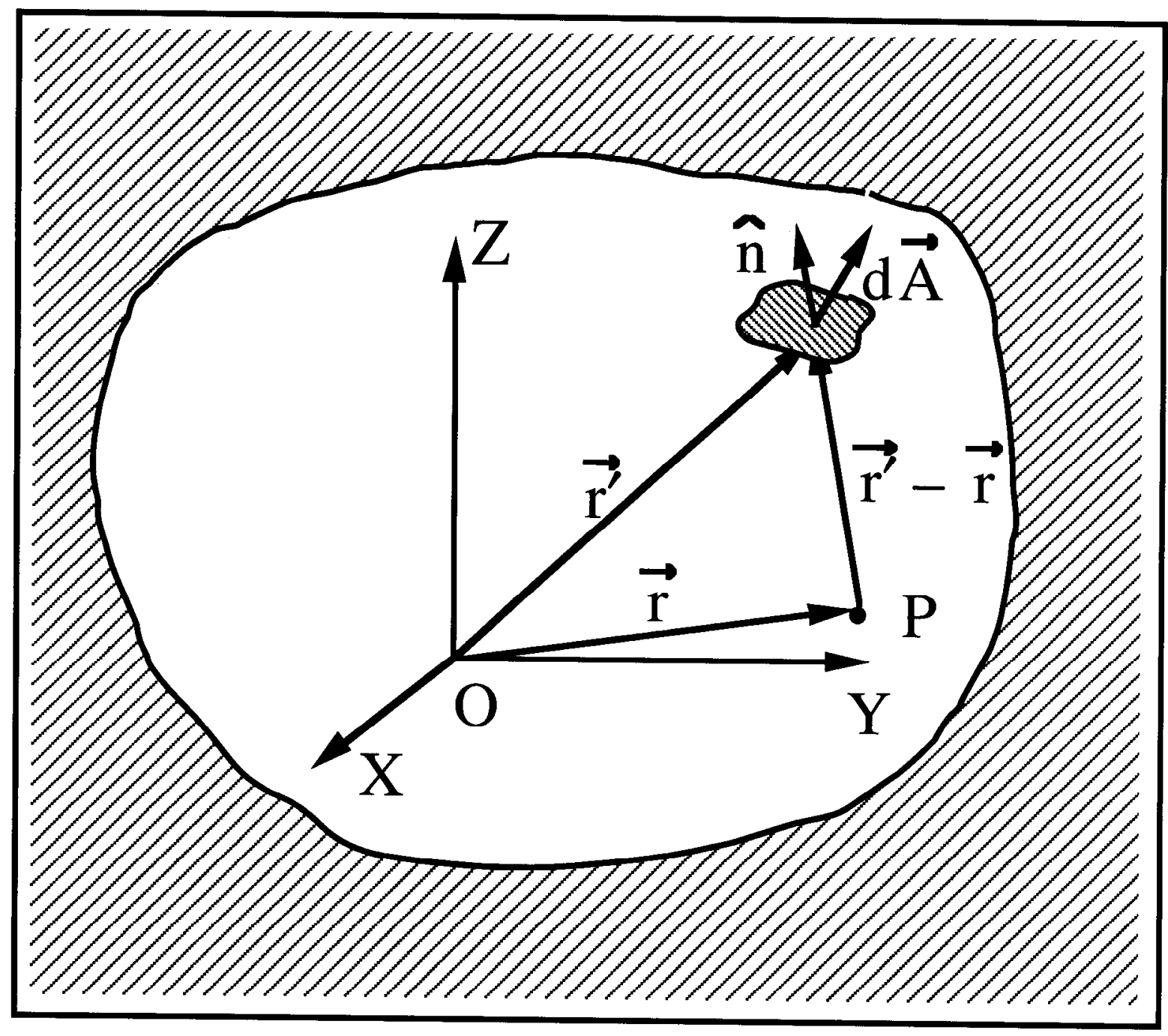

Figure 1 


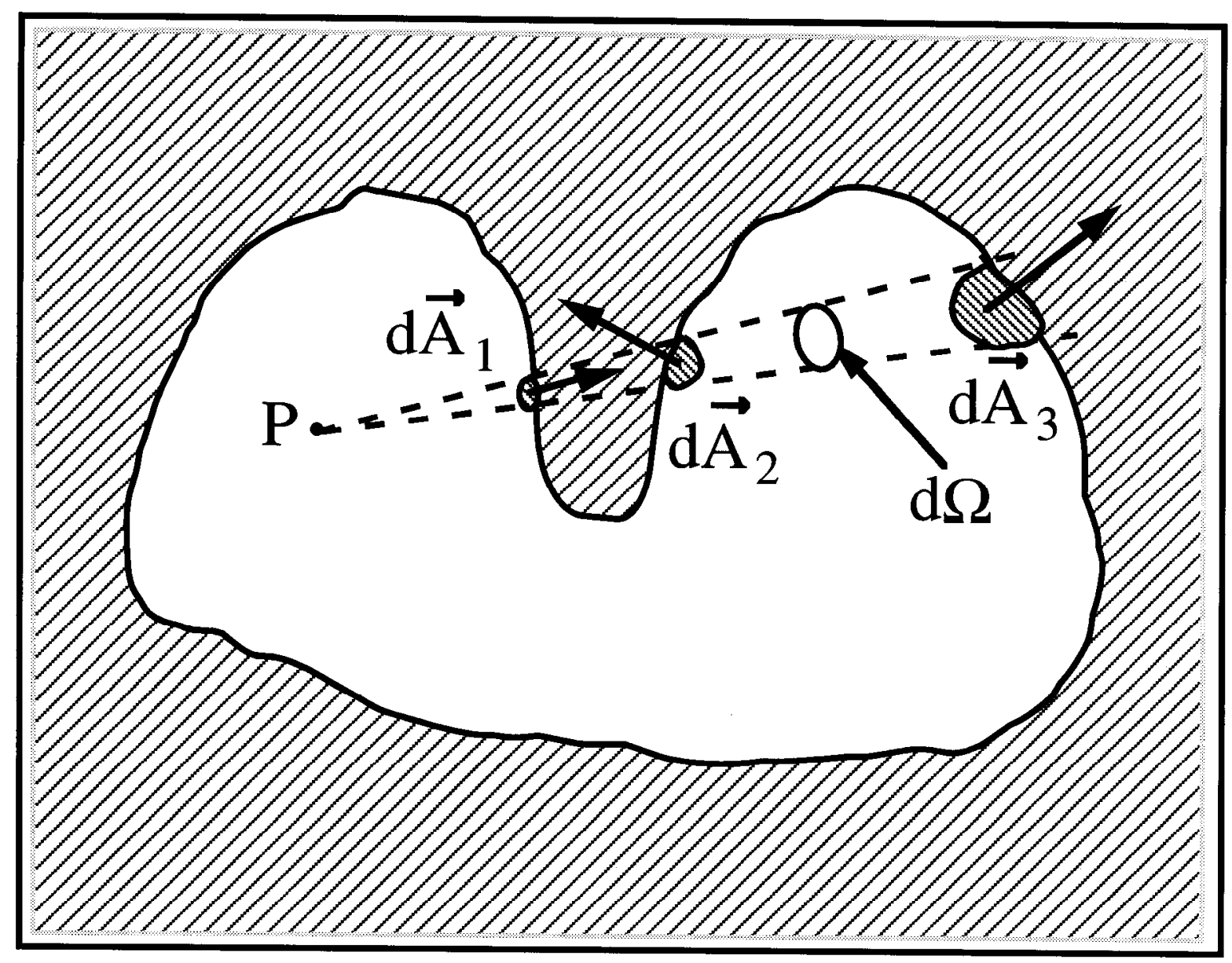

Figure 2 